\title{
Referenciação oftalmológica em idade pediátrica: estudo retrospetivo de doze meses consecutivos de referenciação oftalmológica hospitalar
}

Inês Alves Casal, ${ }^{1}$ Sílvia Monteiro, ${ }^{1}$ Ana Figueiredo, ${ }^{1}$ Carolina Vale, ${ }^{1}$ Tânia Borges, ${ }^{1}$ Vasco Miranda, ${ }^{1-2}$ Ricardo Parreira,,${ }^{1-2}$ Pedro Menéres, ${ }^{1-2}$

\begin{abstract}
RESUMO
O objetivo deste estudo foi avaliar o estado da referenciação de crianças à especialidade hospitalar de oftalmologia, no Centro Hospitalar do Porto, analisando os motivos de referenciação, patologias diagnosticadas, eficácia dessa referenciação e o impacto visual nas crianças.

Método: Estudo retrospetivo e descritivo dos processos clínicos das crianças com idade menor ou igual a 10 anos, referenciadas à consulta de oftalmologia pediátrica entre julho de 2011 e julho de 2012.

Resultados: Um total de 649 crianças foi referenciado, tendo sido os motivos mais frequentes a suspeita de estrabismo em $41,9 \%$ dos casos, rastreio de patologia oftalmológica em $22,6 \%$ e suspeita de baixa acuidade visual (AV) em $15,2 \%$. Das crianças referenciadas por suspeita de estrabismo confirmou-se o diagnóstico em $40,8 \%$ dos casos (média das idades $4,5 \pm 2,2$ anos), sendo que $16,8 \%$ das crianças referenciadas por outros motivos apresentavam também estrabismo. No total, $62,3 \%$ foram classificados como endotropias e 32,3\% como exotropias. Das crianças referenciadas por suspeita de baixa AV, confirmou-se o diagnóstico em $39 \%$ dos casos e a principal causa de diminuição da AV foram as ametropias ( $52 \%$ dos casos). Das crianças referenciadas por outros motivos, 13,3\% apresentavam diminuição mensurável da AV e $20 \%$ tinham ametropias potencialmente ambliogénicas. Apenas $40 \%$ das crianças referenciadas por suspeita de baixa AV apresentavam registo de AV no pedido de referenciação. Foi diagnosticada ambliopia em $20,1 \%$ de todas as crianças observadas.

Discussão: Os problemas que mais frequentemente motivaram a referenciação para oftalmologia pediátrica foram a suspeita de estrabismo e de diminuição da AV. No entanto, confirmou-se o diagnóstico em menos de metade das crianças referenciadas e uma percentagem significativa dos casos diagnosticados foi referenciado por outro motivo. A média das idades de diagnóstico de estrabismo foi tardia e a percentagem de ambliopia elevada. Este estudo reforça a importância da realização do exame oftalmológico de rastreio nos cuidados de saúde primários e o seu impacto na referenciação, diagnóstico e tratamento atempado destas patologias em idade pediátrica.
\end{abstract}

Palavras-chave: Referenciação; Estrabismo; Ametropia; Ambliopia

\section{INTRODUÇÃO}

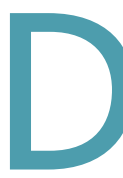

e acordo com a Organização Mundial da Saúde, os erros refrativos (ametropias) não corrigidos são a segunda maior causa de diminuição da acuidade visual (AV) em todas as idades, géneros e etnias. ${ }^{1}$ Há cerca de 153 milhões de casos de deficiência visual em todo o mundo, com 12,8 milhões de crianças (dos 5 aos 15 anos de idade) afeta- das por erros refrativos (ametropias) não corrigidos, ${ }^{1}$ tanto em países desenvolvidos como em desenvolvimento, ${ }^{2}$ sendo este um importante problema de saúde pública. $^{3-4}$

\footnotetext{
1. Centro Hospitalar do Porto, Hospital Geral de Santo António.

2. Instituto de Ciências Biomédicas Abel Salazar, Universidade do Porto.
} 
A população pediátrica é particularmente vulnerável, não só porque a maioria das ametropias surge nesta faixa etária mas também porque a deteção da patologia ocular é mais difícil por múltiplas razões: pela não verbalização das queixas na idade pré-verbal, pela frequente ausência de queixas na idade verbal, pela maior dificuldade na realização e interpretação do exame oftalmológico, etc. A patologia ocular na criança, se não for detetada e tratada atempadamente, pode ter um grande impacto no seu desenvolvimento psicomotor e social, com consequentes dificuldades na aprendizagem escolar e limitações socioeconómicas importantes na idade adulta.

A ambliopia é uma das complicações mais temidas do diagnóstico tardio de patologia oftalmológica na criança e afeta cerca de 0,5 a 3,5\% da população com idade inferior a 8 anos. ${ }^{5-8}$ Define-se como uma diminuição da $\mathrm{AV}$, que não melhora mesmo com correção refrativa num olho estruturalmente sem outras alterações $^{9-10}$ e resulta da ausência de estimulação da retina com imagens nítidas durante o período de desenvolvimento visual (primeiros anos de vida). A ambliopia pode ter uma causa refrativa, estrábica, de privação ou mista. A ambliopia refrativa é consequência do não tratamento de ametropias elevadas (hipermetropia, miopia e astigmatismo), tanto bilaterais como unilaterais (anisometropia), constituindo a ambliopia refrativa a segunda causa de cegueira evitável/tratável em idade pediátrica em países desenvolvidos. ${ }^{5-7} \mathrm{~A}$ ambliopia estrábica resulta de alterações no alinhamento ocular (estrabismo) e subsequente interrupção da visão binocular, com privilégio da visão de um olho e supressão da visão do outro. A ambliopia de privação é o subtipo mais grave e que necessita de uma intervenção mais precoce, sendo consequência da diminuição ou ausência de estimulação luminosa da retina por opacidade dos meios oculares (e.g., catarata, cicatrizes corneanas) ou patologia das pálpebras (e.g., ptose, neoformações). Em todos os casos, a atuação precoce é fundamental, sendo o sucesso dependente da causa, do grau de gravidade da ambliopia e da idade em que se inicia o tratamento. A idade a partir da qual já não é possível tratar uma ambliopia é debatível. Há casos descritos de melhoria com tratamentos instituídos já durante a adolescência e há casos que não melhoram significativamente mesmo com instituição de tratamento aos 5-6 anos. É razoavelmente consensual que quanto mais desfocada for a imagem retiniana mais rápida será a instalação da ambliopia e maior será a sua gravidade. Igualmente, quanto mais grave for a ambliopia, mais precoce tem que ser o tratamento para ser bem-sucedido, sendo que após os oito anos (idade em que o desenvolvimento visual geralmente já está finalizado) o tratamento é na maioria dos casos ineficaz. ${ }^{11-13} \mathrm{O}$ estrabismo é também uma das patologias oculares mais frequentes em idade pediátrica. Define-se como uma alteração no alinhamento ocular, afetando cerca de 3-5\% de todas as crianças no mundo. ${ }^{14-19}$ Está frequentemente associado a várias patologias sistémicas como as síndromas crânio-sinostóticas $(43,3 \%)^{20}$ e a síndroma de Down (26,1\%); ${ }^{21,23} \mathrm{e} \mathrm{também} \mathrm{é} \mathrm{frequente} \mathrm{em} \mathrm{indivíduos}$ com QI baixo (15,8\%), epilepsia e em doentes com paralisia cerebral $(39 \%) .{ }^{22-26}$ Está também descrito que crianças com estrabismo têm um risco de desenvolver patologia psiquiátrica em idade adulta 2,6 vezes superior às crianças sem estrabismo ${ }^{18} \mathrm{e}$ estudos de qualidade de vida mostraram resultados menos satisfatórios entre os doentes com estrabismo. ${ }^{19,27}$ A melhor forma de identificar patologia ocular atempadamente e assim evitar a diminuição irreversível de acuidade visual é a realização de rastreios oftalmológicos regulares e, perante a suspeita de alguma patologia ou fatores de risco, a articulação e referenciação da criança a uma consulta de oftalmologia. No Sistema Nacional de Saúde (SNS) Português várias estratégias multidisciplinares têm sido propostas nas últimas décadas para melhorar a eficácia desta referenciação. ${ }^{28-30} \mathrm{O}$ Programa Nacional para a Saúde da Visão, ${ }^{29}$ da Direção-Geral da Saúde, recomenda que todas as crianças saudáveis sejam submetidas a um exame visual sumário ao nascimento, aos dois e seis meses e aos dois, cinco e dez anos de idade, a realizar pelo médico de medicina geral e familiar (MGF). Em caso de anomalia ou dúvida está recomendada a referenciação para uma consulta hospitalar de oftalmologia. Em crianças com alto risco de patologia oftalmológica poderá estar indicada uma referenciação hospitalar precoce mesmo na ausência de sinais/sintomas oftalmológicos.

O objetivo deste estudo foi avaliar o estado atual da referenciação de crianças à especialidade hospitalar de oftalmologia, no Centro Hospitalar do Porto, analisando os motivos de referenciação, as patologias diagnos- 


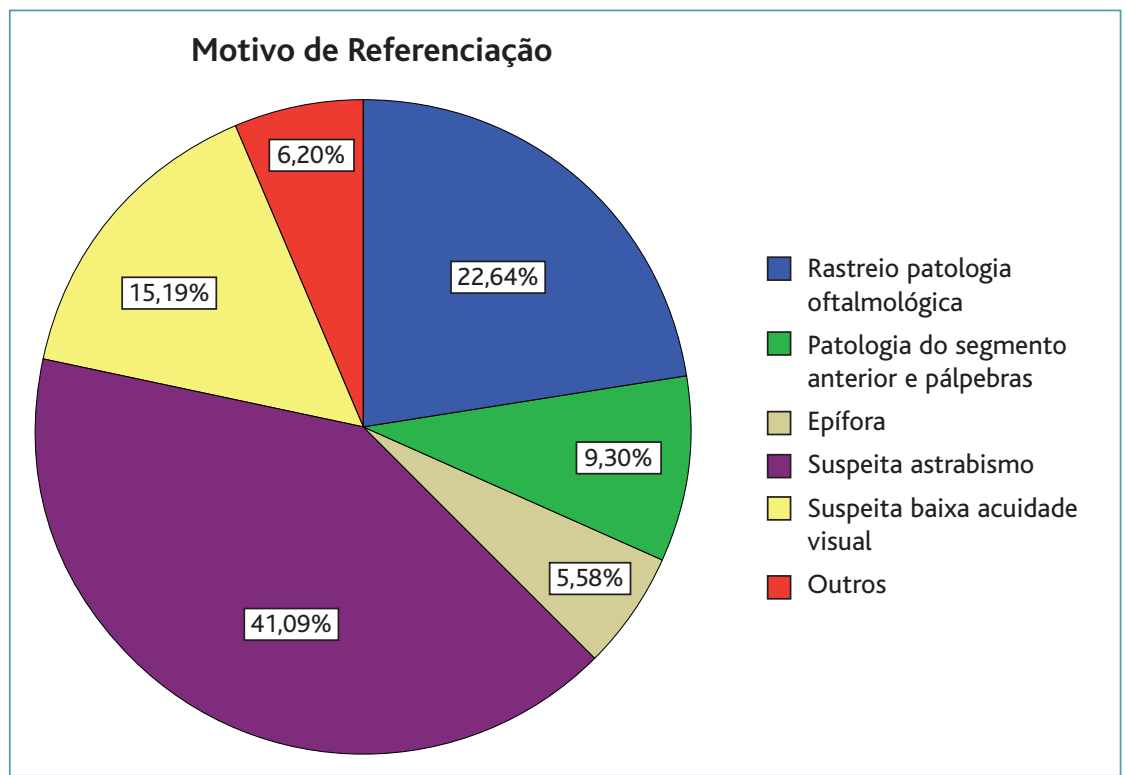

Figura 1. Motivos de referenciação à consulta de oftalmologia.

ticadas, a eficácia dessa referenciação e o seu impacto visual nas crianças.

\section{MÉTODO}

Foi realizado um estudo retrospetivo das crianças com idade igual ou inferior a dez anos, referenciadas à consulta de oftalmologia pediátrica do Centro Hospitalar do Porto durante doze meses consecutivos (entre julho de 2011 e julho de 2012). Foram excluídos os processos das crianças que faltaram à primeira ou às consultas subsequentes.

A amostra foi caracterizada quanto à idade da criança, sexo, motivo de referenciação, especialidade do médico referenciador, suspeita de patologia oftalmológica por parte dos pais, presença/ausência de erros refrativos e tipo de erro refrativo, presença/ausência de erro refrativo potencialmente ambliogénico; presença de estrabismo e tipo de estrabismo; presença de ambliopia e tratamentos efetuados e patologia oftalmológica e não oftalmológica associada. Foi considerada como ametropia relevante para este estudo um erro refrativo com equivalente esférico $\geq 0,75$ dioptrias. Os valores utilizados pela Academia Americana de Oftalmologia foram utilizados como referência para classificar ametropias potencialmente ambliogénicas. Foi considerada baixa AV quando esta era inferior ou igual a 0,6 (escala decimal) nas crianças com idade menor que cinco anos; e quando a AV era inferior a 0,8 nas crianças com idade igual ou superior a cinco anos.

A definição e a classificação da ambliopia foram realizadas segundo o Pediatric Eye Disease Investigation Group (PEDIG). Nas crianças pré-verbais, em que não foi possível obter um registo de $\mathrm{AV}$, considerou-se diagnóstico de presunção de ambliopia se houvesse uma preferência de fixação mesmo após correção refrativa adequada.

A análise estatística foi realizada usando o SPSS ${ }^{\circledR}$ (v. 22). Foi utilizado o teste $t$-student para diferenças entre variáveis paramétricas contínuas; Qui-quadrado e teste exato de Fisher para diferenças entre variáveis categóricas; o valor de $p<0,05$ foi considerado estatisticamente significativo.

\section{RESULTADOS}

Entre julho de 2011 e julho de 2012, 649 crianças com menos de 10 anos de idade foram referenciadas à consulta de oftalmologia pediátrica do Centro Hospitalar do Porto: $41,9 \%$ foram referenciadas por suspeita de estrabismo, $22,6 \%$ para rastreio de patologia oftalmológica, $15,2 \%$ por suspeita de baixa $A V, 9,3 \%$ por suspeita de patologia do segmento anterior e pálpebras, 5,6\% por epífora e $6,2 \%$ por outros motivos (Figura 1). Os motivos de referenciação por especialidade referenciadora são apresentados no Quadro I.

De todas as crianças referenciadas foi detetada patologia oftalmológica em $46,4 \%$ dos casos, mas só em $46,7 \%$ das crianças é que a patologia detetada correspondeu ao motivo de referenciação. A distribuição dos motivos de referenciação e dos diagnósticos por idade são apresentados nas Figuras 2 e 3.

\section{Suspeita de estrabismo}

Das crianças referenciadas por suspeita de estrabismo confirmou-se o diagnóstico em $40,8 \%$ dos casos (em $36 \%$ das crianças referenciadas por MGF, em 39,6\% 


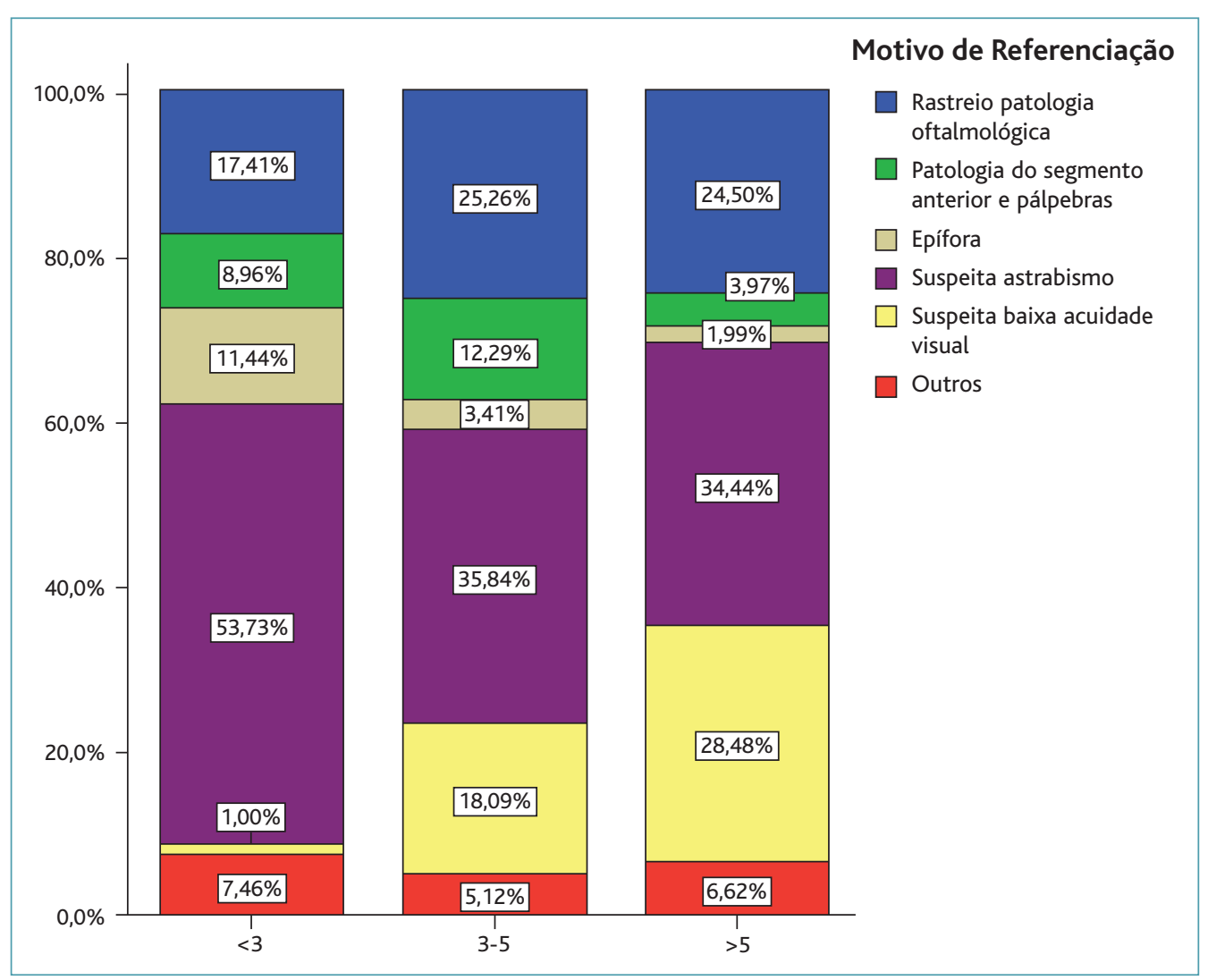

Figura 2. Distribuição dos motivos de referenciação por idade.

das referenciadas por pediatria e em $73,3 \%$ das referenciadas por oftalmologia). No entanto, $16,8 \%$ das crianças referenciadas por outros motivos apresentavam também estrabismo. No total diagnosticou-se estrabismo em 130 crianças $(20 \%$ de todas as observadas).

A média das idades aquando do diagnóstico de estrabismo foi de 4,5 $\pm 2,2$ (0,3-9) anos, sendo $50,8 \%$ do sexo feminino. No total, $62,3 \%$ dos estrabismos diagnosticados foram classificados como endotropias, $32,3 \%$ como exotropias e em $5,4 \%$ foram diagnosticados outros estrabismos. A média da idade aquando do diagnóstico das crianças com endotropias foi superior à das crianças com exotropias, mas essa diferença não apresentou significado estatístico $(4,6 \pm 2,1$ vs 4,3 $\pm 2,3$; $p=0,4)$.

De notar que as crianças com estrabismo apresentaram maior associação a patologia neurocognitiva $(32,3 \%)$ do que os doentes sem diagnóstico de estra- bismo (3,1\%), sendo esta associação mais frequente nas exotropias do que nas endotropias $\left(52,3 \%\right.$ vs $30,1 \%$; $\chi^{2}$ $p<0,001)$.

\section{Suspeita de diminuição da acuidade visual}

A média de idades das crianças referenciadas por

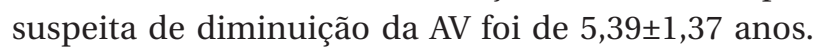
Destas crianças referenciadas por suspeita de baixa AV confirmou-se o diagnóstico em $39 \%$ dos casos. A referenciação foi feita em $71,3 \%$ das crianças referenciadas por MGF, em 25,5\% das referenciadas por pediatria e em $2,1 \%$ das referenciadas por oftalmologia; em $19,5 \%$ dos casos havia também suspeita por parte dos pais. A diminuição da AV foi unilateral em 9,4\% dos casos. A principal causa de diminuição da AV foram as ametropias em $52 \%$ dos casos, seguida da ambliopia estrábica e em menor percentagem de patologia retiniana e do nervo ótico. Das crianças referenciadas por suspeita de baixa $\mathrm{AV}$ em que não foi possível quantificar a $\mathrm{AV}(42,9 \%)$, 


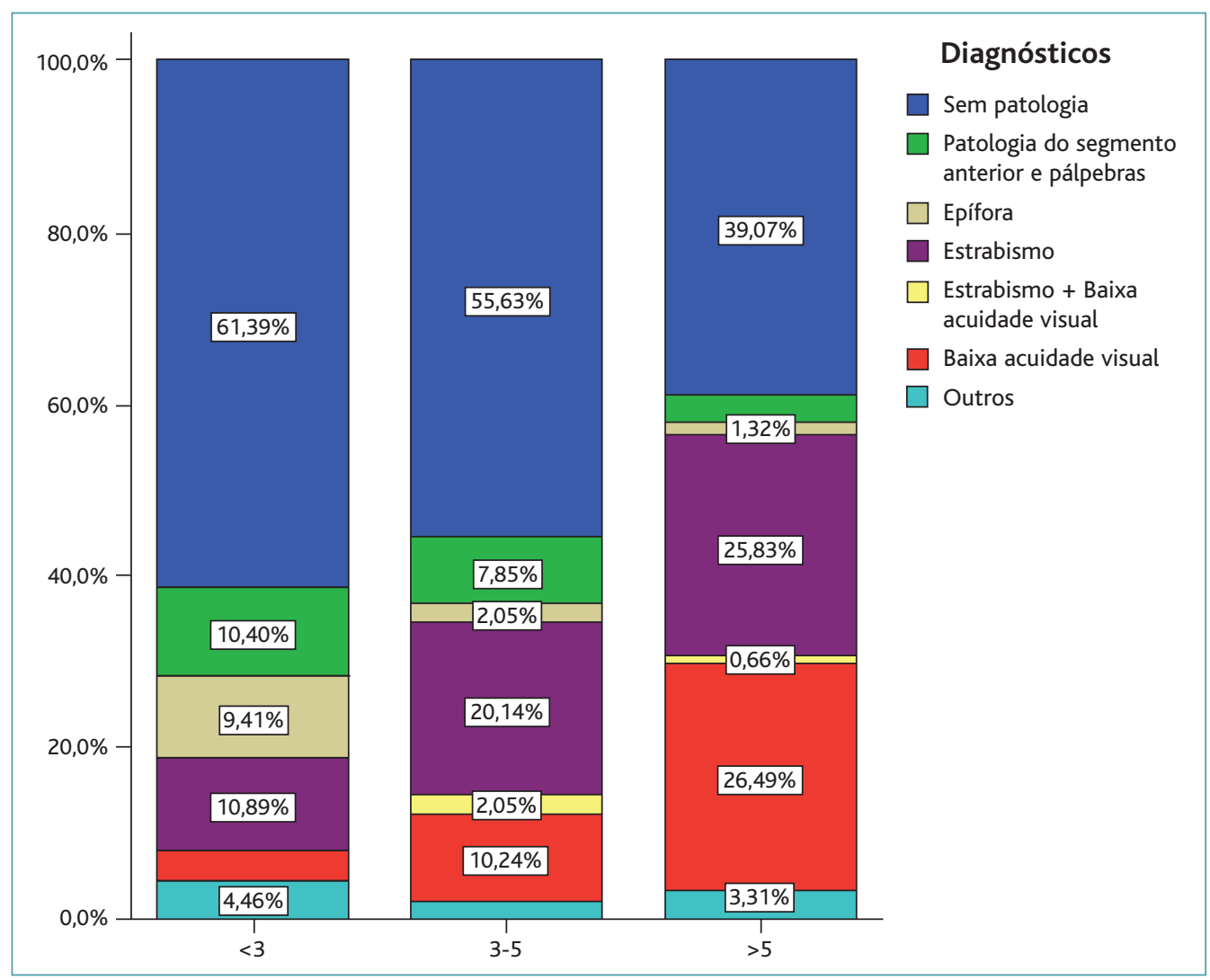

Figura 3. Distribuição dos diagnósticos por idade.

$35,7 \%$ apresentavam ametropias potencialmente ambliogénicas. De todas as crianças referenciadas por outros motivos 13,3\% também apresentavam diminuição mensurável da AV e $20 \%$ tinham ametropias potencialmente ambliogénicas. Apenas $40 \%$ das crianças referenciadas por suspeita de baixa $\mathrm{AV}$ apresentavam registo de $\mathrm{AV}$ no pedido de referenciação. Das restantes $60 \%, 2 \%$ tinham idade inferior a três anos, $50 \%$ tinham idades compreendidas entre os 3-5 anos e $47 \%$ idade igual ou superior a seis anos.

\section{Ametropias}

De todas as crianças avaliadas $39,6 \%$ apresentava uma ametropia relevante. A média das idades das crianças com ametropias relevantes era de 4,3 $\pm 2,1$ anos e $52,9 \%$ eram do sexo masculino. O erro refrativo mais frequente foi o astigmatismo hipermetrópico composto $(47,5 \%)$, seguido de hipermetropia $(21 \%)$, astigmatismo miópico composto $(9,7 \%)$, astigmatismo hiper- metrópico simples $(7,8 \%)$, astigmatismo misto $(7,4 \%)$, astigmatismo miópico simples $(5,1 \%)$ e miopia $(1,6 \%)$ Anisometropias potencialmente ambliogénicas foram observadas em 10,5\% dos casos. Das crianças com estrabismo $80,8 \%$ tinham ametropias com equivalente esférico superior a 0,75 dioptrias, $26,9 \%$ tinham ametropias com equivalente esférico superior a quatro dioptrias e 10,8\% tinham anisometropias com diferença de equivalentes esféricos superior a 1,5 dioptrias. Os erros refrativos mais frequentes nas crianças com estrabismo foram o astigmatismo composto hipermetrópico $(43,8 \%)$ e a hipermetropia $(23,1 \%)$. Os estrabismos mais frequentemente associados a equivalentes esféricos superiores a quatro dioptrias foram a endotropia refrativa (60\% das endotropias refrativas) e a endotropia parcialmente acomodativa ( $52,2 \%$ das endotropias parcialmente acomodativas). As crianças com patologia neurocognitiva tinham mais frequentemente ametropias potencialmente ambliogénicas do que 
as crianças saudáveis $(48,5 \%$ vs $\left.18,8 \% ; \chi^{2}=31,56 p<0,001\right)$. Das crianças com ametropias relevantes só se considerou necessária a prescrição de óculos em 54,3\%.

\section{Ambliopia}

Foi diagnosticada ambliopia em $20,1 \%$ de todas as crianças observadas: $33,3 \%$ de causa refrativa, $16,3 \%$ de causa estrábica e 45,9\% de causa mista. As ametropias mais frequentemente associadas à ambliopia refrativa foram o astigmatismo hipermetrópico composto (52,3\%), seguido do astigmatismo misto (15,9\%) ( $\mathrm{F}=25,96 p<0,01)$. A ambliopia puramente estrábica apresentou-se mais frequentemente associada às exotropias $(70,4 \%)$ do que às endotropias $(18,5 \%)(\mathrm{F}=47,96 ; p<0,001)$. Das crianças pré-verbais, em que não foi possível testar AV, 47,1\% apresentavam fixação preferencial de um olho, sugerindo também uma possível ambliopia associada.

\section{DISCUSSÃO}

A suspeita de estrabismo foi a causa mais frequente de referenciação de crianças com idade inferior a 10 anos à consulta de oftalmologia pediátrica (mais de $40 \%$ ), demonstrando a importância da suspeita de estrabismo no SNS nacional.

A prevalência dos diferentes tipos de estrabismo é variável nos vários estudos populacionais realizados. ${ }^{14-16}$ No presente estudo, a frequência dos estrabismos convergentes foi quase o dobro da frequência dos estrabismos divergentes. Não sendo este um estudo populacional, esta assimetria pode representar uma maior dificuldade dos médicos referenciadores em detetar estrabismos divergentes.

Neste estudo, o valor preditivo positivo das referenciações por suspeita de estrabismo foi relativamente baixo (40,8\%), havendo uma percentagem relevante de crianças com estrabismo que foram referenciadas à consulta por outros motivos, sem suspeita prévia da patologia (16,8\%). A sensibilidade global da referenciação por suspeita de estrabismo foi de $83,8 \%$ e a especificidade de $73,6 \%$. No entanto, o facto de este estudo não ser um estudo populacional e de terem sido incluídas crianças com idades muito superiores à da idade provável de início do estrabismo torna estes valores de sensibilidade e especificidade artificialmente elevados e não comparáveis aos valores de estudos de rastreio populacional. Relativamente à ambliopia estrábica, a frequência encontrada no presente estudo foi superior à descrita noutros estudos ${ }^{14}(33 \%$ vs $12,5 \%)$, sendo que a idade tardia de diagnóstico de estrabismo (média de 4,5 $\pm 2,2$ anos) provavelmente contribuiu para esta disparidade; o facto de muitas destas crianças terem patologia neurocognitiva associada, constituindo um grupo de pior prognóstico comparativamente com estudos populacionais, poderá também contribuir para esta diferença. Os estrabismos mais frequentemente associados a ambliopia foram a endotropia parcialmente acomodativa $(91,3 \%)$ e a exotropia infantil $(87,5 \%)$.

Do total de crianças referenciadas por suspeita de baixa $\mathrm{AV}$, apenas $39 \%$ apresentava realmente uma diminuição da AV, havendo uma percentagem significativa de crianças com baixa AV que foram referenciadas à consulta por outros motivos (13,3\%). Destas, apenas $40 \%$ apresentavam a avaliação da AV no pedido de re- 
ferenciação. Das crianças em que não havia registo de medição da AV, 98\% encontravam-se em idade verbal. Esta alta percentagem pode refletir a dificuldade na avaliação da AV por não existência de local e material apropriado ou por falta de tempo para a sua realização.

A percentagem de ametropias diagnosticadas nas crianças referenciadas por suspeita de baixa AV foi de $52 \%$, em comparação com os $39 \%$ encontrados nas crianças referenciadas para rastreio de patologia oftalmológica. De todas as crianças com ametropias, só foi considerada necessária prescrição de óculos em 54,3\%.

Atualmente existem múltiplos aparelhos de fotorefração portáteis especialmente desenhados para rastreio de ametropias em idade pediátrica. Estes aparelhos permitem um exame não invasivo e extremamente rápido (segundos) que deteta erros refrativos potencialmente ambliogénicos com uma sensibilidade de 78-98\% e uma especificidade de $59-88 \%,{ }^{31-32}$ sendo particularmente úteis no rastreio de crianças com menos de três anos de idade. Estes exames podem ser efetuados por técnicos devidamente treinados e têm sido instituídos com sucesso em esquemas de rastreio oftalmológico em múltiplos países, constituindo um método eficaz no complemento dos atuais protocolos de identificação de crianças em risco de patologia ocular e que necessitam de referenciação para consulta oftalmológica.

\section{CONCLUSÃO}

Este estudo revelou que menos de metade das crianças referenciadas à consulta hospitalar de oftalmologia em idade ambliogénica tinha patologia ocular. Adicionalmente observou-se que, entre as crianças com patologia, mais de metade apresentava pelo menos uma patologia da qual não havia suspeita aquando da referenciação.

A média da idade do diagnóstico de estrabismo (4,5 anos) e de ametropia relevante (4,3 anos) foi elevada, estando assim associadas a uma alta percentagem de ambliopia, o que poderia provavelmente ser minorado através da deteção e tratamento mais precoces.

O facto de $60 \%$ das referenciações hospitalares por suspeita de diminuição de AV não se acompanharem de registos de acuidades visuais suscita questões importantes: quais as dificuldades encontradas em obter essas avaliações e se essas dificuldades também com- prometem o cumprimento adequado do restante rastreio oftalmológico em cuidados de saúde primários. $\mathrm{Na}$ opinião dos autores, o Programa Nacional para a Saúde da Visão, que já não é revisto desde 2008, poderá beneficiar de uma simplificação que se coadune com a realidade da prática clínica atual dos médicos de MGF e de pediatria. Adicionalmente, perante os resultados encontrados, seria relevante a realização de um projeto-piloto para avaliar a utilidade prática e viabilidade da inclusão no SNS de um programa de rastreio oftalmológico por fotorefração em crianças pré-verbais.

Os cuidados oftalmológicos de saúde em idade pediátrica prestados no SNS Português integram, como primeiro elemento, o médico de MGF e o pediatra. A eficácia dos cuidados prestados está assim intimamente ligada à sua capacidade de detetar e referenciar e à capacidade do oftalmologista para avaliar, diagnosticar e tratar atempadamente. Este circuito completa-se devolvendo informação clínica adequada ao médico referenciador. Este estudo reforça a importância da prática do exame oftalmológico de rastreio nestas especialidades e a necessidade de melhorar e manter a formação contínua nesta área.

\section{REFERÊNCIAS BIBLIOGRÁFICAS}

1. Barry JC, König HH. Test characteristics of orthoptic screening examination in 3 year old kindergarten children. Br J Ophthalmol. 2003;87(7): 909-16.

2. Quinn GE, Berlin JA, James M. The Teller acuity card procedure: three testers in a clinical setting. Ophthalmology. 1993;100(4):488-94.

3. Sousa PS, Laranjeira JA, Ferreira E, Queiroz L, Doria JL. A visão em desenvolvimento infantil: o programa de saúde da visão na Fundação Nossa Senhora do Bom Sucesso. Lisboa: Fundação Nossa Senhora do Bom Sucesso; 2001. ISBN 9789729862519

4. Klaeger C, Munier A. Ophthalmological tips and tricks for the pediatricians. Paediatrica. 2003;1:1-7.

5. Resnikoff S, Pascolini D, Mariotti SP, Pokharel GP. Global magnitude of visual impairment caused by uncorrected refractive errors in 2004. Bull World Health Organ. 2008;86(1):63-70.

6. Matsumura $\mathrm{H}, \mathrm{Hirai} \mathrm{H}$. Prevalence of myopia and refractive changes in students from 3 to 17 years of age. Surv Ophthalmol. 1999;44 Suppl 1:S109-15.

7. Thylefors B. A global initiative for the elimination of avoidable blindness. Am J Ophthalmol. 1998;125(1):90-3.

8. Dandona R, Dandona L. Refractive error blindness. Bull World Health Organ. 2001;79(3):237-43.

9. Friedmann L, Biedner B, David R, Sachs U. Screening for refractive errors, strabismus and other ocular anomalies from ages 6 months to 3 years. J Pediatr Ophthalmol Strabismus. 1980;17(5):315-7.

10. Friedman Z, Neumann E, Hyams SW, Peleg B. Ophthalmic screening of 
38,000 children, age 1 to $21 / 2$ years, in child welfare clinics. J Pediatr Ophthalmol Strabismus. 1980;17(4):261-7.

11. Stayte M, Johnson A, Wortham C. Ocular and visual defects in a geographically defined population of 2-year-old children. $\mathrm{Br} J \mathrm{Ophthalmol}$. 1990;74(8):465-8.

12. Williams C, Harrad RA, Harvey I, Sparrow JM. Screening for amblyopia in preschool children: results of a population-based, randomised controlled trial: ALSPAC study team, Avon Longitudinal Study of Pregnancy and Childhood. Ophthalmic Epidemiol. 2001;8(5):279-95.

13. US Committee on Preschool Vision Screening. Preschool vision screening guidelines: revised 2000 [Internet]. US Committee on Preschool Vision Screening; 2000. Available from: http://www.madriver.k12.oh.us/ health/Documents/Preschool\%20vision\%20.pdf

14. Chia A, Dirani M, Chan YH, Gazzard G, Au Eong KG, Selvaraj P, et al. Prevalence of amblyopia and strabismus in young singaporean chinese children. Invest Ophthalmol Vis Sci. 2010;51(7):3411-7.

15. Graham PA. Epidemiology of strabismus. Br J Ophthalmol. 1974;58(3): 224-31.

16. Greenberg AE, Mohney BG, Diehl NN, Burke JP. Incidence and types of childhood esotropia: a population-based study. Ophthalmology. 2007; 114(1):170-4.

17. Govindan M, Mohney BG, Diehl NN, Burke JP. Incidence and types of childhood exotropia: a population-based study. Ophthalmology. 2005; 112(1):104-8.

18. Olson JH, Louwagie CR, Diehl NN, Mohney BG. Congenital esotropia and the risk of mental illness by early adulthood. Ophthalmology. 2012; 119(1):145-9.

19. Tollefson MM, Mohney BG, Diehl NN, Burke JP. Incidence and types of childhood hypertropia: a population-based study. Ophthalmology. 2006;113(7):1142-5.

20. Tay T, Martin F, Rowe N, Johnson K, Poole M, Tan K, et al. Prevalence and causes of visual impairment in craniosynostotic syndromes. Clin Exp Ophthalmol. 2006;34(5):434-40.

21. Stirn Kranjc B. Ocular abnormalities and systemic disease in Down syndrome. Strabismus. 2012;20(2):74-7.

22. Gogate P, Soneji FR, Kharat J, Dulera H, Deshpande M, Gilbert C. Ocular disorders in children with learning disabilities in special education schools of Pune, India. Indian J Ophthalmol. 2011;59(3):223-8.

23. Mohney BG, McKenzie JA, Capo JA, Nusz KJ, Mrazek D, Diehl NN. Men- tal illness in young adults who had strabismus as children. Pediatrics. 2008; 122(5):1033-8.

24. Schiffman J, Maeda JA, Hayashi K, Michelsen N, Sorensen HJ, Ekstrom $M$, et al. Premorbid childhood ocular alignment abnormalities and adult schizophrenia-spectrum disorder. Schizophr Res. 2006;81(2-3):253-60.

25. Toyota T, Yoshitsugu K, Ebihara M, Yamada K, Ohba H, Fukasawa M, et al. Association between schizophrenia with ocular misalignment and polyalanine length variation in PMX2B. Hum Mol Genet. 2004;13(5):551-61.

26. Van Splunder J, Stilma JS, Bernsen RM, Evenhuis HM. Prevalence of ocular diagnoses found on screening 1539 adults with intellectual disabilities. Ophthalmology. 2004;111(8):1457-63.

27. Singman E, Matta N, Tian J, Silbert D. The accuracy of the plusoptiX for measuring pupillary distance. Strabismus. 2014;22(1):21-5.

28. Pinto F, Guerra I, Maia I, Rodrigues S. Rastreio oftalmológico infantil nos cuidados primários [Paediatric ophthalmologic screening in primary healthcare]. Acta Pediatr Port. 2007;38(3):99-102. Portuguese

29. Direção-Geral da Saúde. Programa nacional para a saúde da visão: circular normativa n. ${ }^{\circ}$ 02/DGCG, de 17/03/2005. Lisboa: DGS; 2005.

30. Santos IM, Coelho I, Rosário F, Machado P, Nery L, Ribeiro J, et al. Referenciação aos cuidados de saúde secundários em idade pediátrica [Referral to secondary pediatric care]. Rev Port Clin Geral. 2011;27(5): 422-32. Portuguese

31. Peterseim MM, Papa CE, Wilson ME, Cheeseman EW, Wolf BJ, Davidson JD, et al. Photoscreeners in the pediatric eye office: compared testability and refractions on high-risk children. Am J Ophthalmol. 2014;158 (5):932-8.

32. Silbert D, Matta N, Tian J, Singman E. Comparing the SureSight autorefractor and the plusoptiX photoscreener for pediatric vision screening. Strabismus. 2014;22(2):64-7.

\section{CONFLITO DE INTERESSES}

Os autores declaram não ter quaisquer conflitos de interesse.

\section{ENDEREÇO PARA CORRESPONDÊNCIA}

Inês Alves Casal

E-mail: inesacasal@gmail.com

Recebido em 13-01-2016

Aceite para publicação em 11-02-2018 


\section{ABSTRACT}

\section{REFERRAL TO PEDIATRIC OPHTHALMOLOGY: RETROSPECTIVE STUDY OF TWELVE MONTHS OF REFERRALS}

The aim of this study was to evaluate the current state of the referral of children to the specialty of Ophthalmology at our Hospital-Centro Hospitalar do Porto, analyzing the reasons for referral, diagnosed diseases, effectiveness of referral and the visual impact on children.

Methods: Retrospective and descriptive study of medical records of children aged $\leq 10$ years-old referred to the Pediatric Ophthalmology Clinic, between July 2011 and July 2012.

Results: A total of 649 children were referred and the most frequent reasons included suspected strabismus in $41.9 \%$, screening for ocular pathology in $22.6 \%$, and suspected low visual acuity (VA) in $15.2 \%$ of the cases. Amongst children referred for suspected strabismus, the diagnosis was confirmed in $40.8 \%$ of the cases (mean age of $4.5 \pm 2.2$ years); $16.8 \%$ of children referred for other reasons also had strabismus. $62.3 \%$ were classified as esotropias and $32.3 \%$ as exotropias. Amongst the children referred for suspected low VA, the diagnosis was confirmed in $39 \%$ of the cases, and the main cause were ametropias (52\% of cases). Of the children referred for other reasons, $13.3 \%$ had a measurable decrease in AV and $20 \%$ had potentially amblyogenic ametropias. Only $40 \%$ of the children referred by suspected low VA had a VA evaluation in the referral request. Amblyopia was diagnosed in $20.1 \%$ of all children observed.

Discussion: The problems that most frequently motivated referral for pediatric ophthalmology were the suspicion of strabismus and of decreased VA. However, the diagnosis was confirmed in less than half of the referred children, and a significant percentage of diagnosed cases was referred for another reason. The mean age of diagnosis of strabismus was late, and the percentage of amblyopia was high. This study reinforces the importance of conducting ophthalmologic screening in primary health care, and its impact on a timely referral, diagnosis and treatment of these pediatric diseases.

Keywords: Referral; Strabismus; Ametropia; Amblyopia 